\title{
1 Development of Narrow Spectrum ATP-competitive Kinase Inhibitors as Probes for
}

\section{BIKE and AAK1}

3 Rafael M. Couñago ${ }^{1}$, Alison D. Axtman ${ }^{2,3}$, Stephen J. Capuzzi ${ }^{3}$, Hátylas Azevedo ${ }^{4}$, David H.

4 Drewry $^{2,3}$, Jonathan M. Elkins ${ }^{1,5}$, Opher Gileadi ${ }^{1,5}$, Cristiano R. W. Guimarães $^{4}$, Alessandra

5 Mascarello ${ }^{4}$, Ricardo A. M. Serafim ${ }^{1}$, Carrow I. Wells ${ }^{2,3}$, Timothy M. Willson ${ }^{2,3}$, William J.

6 Zuercher ${ }^{2,3, *}$

$7{ }^{1}$ Structural Genomics Consortium, Universidade Estadual de Campinas - UNICAMP,

8 Campinas, SP, Brazil.

$9 \quad{ }^{2}$ Structural Genomics Consortium, UNC Eshelman School of Pharmacy, University of

10 North Carolina at Chapel Hill, Chapel Hill, NC, USA.

$11{ }^{3}$ Division of Chemical Biology and Medicinal Chemistry, UNC Eshelman School of

12 Pharmacy, University of North Carolina at Chapel Hill, Chapel Hill, NC, USA.

$13{ }^{4}$ Aché Laboratórios Farmacêuticos SA, Guarulhos, SP, Brazil.

$14{ }^{5}$ Structural Genomics Consortium and Target Discovery Institute, Nuffield Department of

15 Clinical Medicine, University of Oxford, Old Road Campus Research Building, Oxford,

16 OX3 7DQ, UK.

17 *Correspondence to W. Zuercher, email: william.zuercher@unc.edu 
18 ABSTRACT: Understanding the structural determinants of inhibitor selectivity would

19 facilitate the design and preparation of kinase probes. We describe a pair of matched

20 compounds differing only by one degree of saturation but showing dramatic differential

21 activities at select kinases. We utilized x-ray crystallography and computational analysis

22 to rationalize the basis of the differential activity.

\section{INTRODUCTION}

24 The human protein kinases are a family of over 500 enzymes that are involved in critical

25 aspects of cell signaling but are often dysregulated in disease, especially cancer [1].

26 Protein kinases catalyze the transfer of phosphate from the cofactor ATP to their

27 substrate proteins. Although ATP-competitive protein kinase inhibitors have been

28 successfully developed to treat a range of cancers, inflammatory and fibrotic diseases,

29 the function of a large number of the enzymes remains unknown [2]. Potent and

30 selective small molecule inhibitors are powerful tools to probe the biology of these

31 understudied kinases [3].

32 Because ATP-competitive inhibitors bind to a common active site, they often show

33 activity across multiple kinases. For example, staurosporine inhibits more than 200

34 protein kinases. These broad spectrum inhibitors are useful for initial biochemical

35 characterization but have little utility as probes of kinase biology [4]. By contrast, other

36 ATP-competitive inhibitors possess activity on only a handful of kinases. These narrow

37 spectrum inhibitors have found utility in exploring the function of several historically

38 understudied kinases [5]. 
39 To strengthen the conclusions drawn from use of narrow spectrum kinase inhibitors, a

40 recently established best practice is to use the probe molecule in parallel with a

41 negative control, a closely-related analogue that is inactive for the target kinase [6].

42 Demonstration of divergent results between the probe inhibitor and its negative control

43 increases confidence that the engagement of the target kinase was responsible for the

44 observed biology.

45 For ATP-competitive kinase inhibitors, a systematic approach to develop negative

46 control analogs is to synthesize a molecule that lacks the ability to form the critical hinge

47 binding interaction, typically composed of one or more hydrogen bonds with the highly

48 conserved hinge region in the enzyme [7]. These modifications result in "kinase dead"

49 analogues. Unfortunately, kinase dead analogues often lose affinity not only for the

50 primary target kinase but also for all other off-target kinases. New strategies to create

51 kinase probe negative controls that lose affinity selectively for their target kinase are

52 needed.

53 Here we describe a pair of compounds in which a minor structural change-namely, the

54 inclusion of a cyclopropyl versus an isopropyl substituent proximal to the hinge-binding

55 moiety-leads to unexpectedly large changes in activity within a small subset of protein

56 kinases but no change against many others. We used X-ray crystallography and

57 computational models to study the molecular basis for these differential effects on

58 affinity within the target and off-target kinases in an attempt to define general

59 approaches to design selective negative control analogs. 


\section{RESULTS}

61 As part of a large-scale selectivity screen of ATP-mimetic kinase inhibitors, we identified

62 a pair of very closely related 3-acylaminoindazoles (1 and $\mathbf{2}$ ) that displayed a narrow

63 binding spectrum in the KINOMEscan panel of over 400 human protein kinase assays at

64 an inhibitor concentration of $1 \mu \mathrm{M}$ (S1 Table). Structurally, $\mathbf{1}$ and $\mathbf{2}$ differed only by the

65 addition of two hydrogen atoms in the change from a cyclopropyl to isopropyl

66 carboxamide. 1 and $\mathbf{2}$ had several common kinase targets but also showed some degree

67 of divergence in kinase affinity profiles. A more detailed analysis of their binding

68 characteristics revealed that 1 had submicromolar affinity for 11 kinases and weaker

69 affinity for 2 additional kinases (Table 1), while $\mathbf{2}$ showed submicromolar affinity for

$707 / 11$ of the primary kinase targets and $1 / 2$ of the minor targets. For most of these

71 kinases, only marginal differences in potency were observed between the analogs $(<3-$

72 fold). However, four kinases (AAK1, BIKE, RIOK1, and RIOK3) displayed remarkable

73 differential affinity between the cyclopropyl analog $\mathbf{1}$ and isopropyl analog $\mathbf{2}$ with a

74 preference for the former (27-150-fold).

75 Table 1. $K_{d}$ values for selected kinases. $K_{d}$ values were determined for kinases observed

76 to have $>70 \%$ displacement of immobilized ligand with either $\mathbf{1}$ or $\mathbf{2}$ in a KINOMEscan

77 screen at $1 \mu \mathrm{M}$ compound.

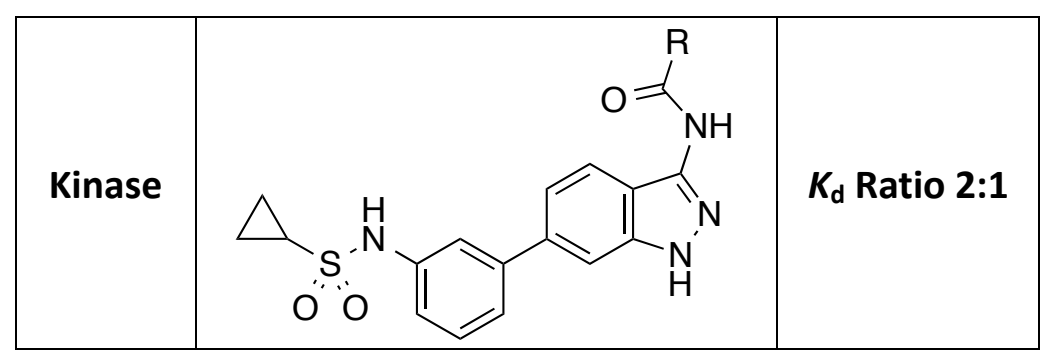




\begin{tabular}{|c|c|c|c|}
\hline & $\begin{array}{c}\mathbf{1}(\mathrm{R}=\text { cyclopropyl) } \\
K_{\mathrm{d}}(\mathrm{nM})\end{array}$ & $\begin{array}{c}\mathbf{2}(\mathrm{R}=i-\mathrm{Pr}) \\
K_{\mathrm{d}}(\mathrm{nM})\end{array}$ & \\
\hline AAK1 & 71 & 8800 & 124 \\
\hline BIKE & 68 & $>10000$ & $>150$ \\
\hline BRAF & 100 & 52 & 0.52 \\
\hline RIOK1 & 170 & 9600 & 56 \\
\hline PDGFRB & 250 & 61 & 0.24 \\
\hline KIT & 290 & 77 & 0.27 \\
\hline RIOK3 & 300 & 8200 & 27 \\
\hline DDR1 & 400 & 150 & 0.38 \\
\hline ABL1 & 540 & 190 & 0.35 \\
\hline RAF1 & 580 & 350 & 0.60 \\
\hline PDGFRA & 590 & 240 & 0.41 \\
\hline CSF1R & 2000 & 1600 & 0.80 \\
\hline P38a & 6900 & 550 & 0.08 \\
\hline
\end{tabular}

79 AAK1 and BIKE, along with the related GAK and STK16, comprise the Numb-associated

80 kinase (NAK) subfamily of protein kinases [8]. NAK family members have been proposed

81 as potential drug targets for a wide range of therapeutic areas, including virology,

82 oncology, neurology, and ophthalmology [9-12]. In contrast, relatively little is known

83 about the relevance to cellular processes of the atypical kinases RIOK1 and RIOK3.

84 Accordingly, to explore the molecular basis of the remarkable differential activities of $\mathbf{1}$

85 and 2, we decided to study their interaction with the NAK family kinases. $\mathbf{1}$ and $\mathbf{2}$ were

86 evaluated in FRET-based ligand binding displacement assays for all four NAK family

87 members [13]. The cyclopropyl analog 1 showed >300-fold higher affinity for AAK1 and

88 BIKE relative to the isopropyl analog 2 (Table 2). Both compounds demonstrated weaker

89 affinity for GAK and STK16, although a >50-fold preference for 1 relative to 2 was

90 observed with the latter kinase. 
91 Table 2. NAK family FRET-based binding displacement assay results.

\begin{tabular}{|c|c|c|c|}
\hline \multirow{2}{*}{ Kinase } & \multicolumn{2}{|c|}{$\boldsymbol{K}_{\mathbf{i}}(\mathbf{n M})$} & \multirow{2}{*}{ Ratio } \\
\cline { 2 - 3 } & $\mathbf{1}$ & $\mathbf{2}$ & \\
\hline AAK1 & 8.3 & 2700 & 320 \\
\hline BIKE & 13 & 3100 & 240 \\
\hline GAK & 1600 & 4900 & 3 \\
\hline STK16 & 330 & 19000 & 58 \\
\hline
\end{tabular}

92

93 Structural analysis of compound 1 binding. BIKE and AAK1 are the closest members

94 within the NAK family and share a high sequence identity-74\% over their kinase

95 domains and $81 \%$ within their ATP-binding sites. In order to obtain insights into the

96 molecular details of ligand interactions with these kinases, we determined the cocrystal

97 structure of BIKE kinase domain bound to compound 1 (Table 3), reasoning that both

98 kinases are likely to interact with $\mathbf{1}$ and $\mathbf{2}$ in similar manners.

99 Table 3. Crystal structure data collection and refinement statistics.

\begin{tabular}{|c|c|}
\hline Protein & BIKE \\
\hline Ligand & 1 \\
\hline X-ray source & DLS 102 \\
\hline $\mathrm{a}(\AA)$ & 41.96 \\
\hline$b(\AA)$ & 111.15 \\
\hline$c(\AA)$ & 164.05 \\
\hline$\alpha, \beta, \gamma\left({ }^{\circ}\right)$ & $90,90,90$ \\
\hline Space group & 1222 \\
\hline Wavelength $(\AA)$ & 0.97949 \\
\hline Resolution limit $(\AA)^{*}$ & $28.6-2.41(2.50-2.41)$ \\
\hline Number of observations* & $81.078(7.855)$ \\
\hline Completeness (\%)* & $99.1(95.2)$ \\
\hline Multiplicity* & $5.3(5.2)$ \\
\hline Rmerge (\%)* & $0.074(0.49)$ \\
\hline 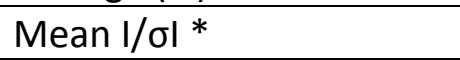 & $13.2(3.1)$ \\
\hline \multicolumn{2}{|l|}{ Refinement } \\
\hline Resolution Range $(\AA ̊)$ & $21.75-2.41$ \\
\hline
\end{tabular}




\begin{tabular}{|l|l|}
\hline Rcryst & 0.176 \\
\hline Rfree & 0.216 \\
\hline No. protein atoms & 2,317 \\
\hline No. ligand atoms & 28 \\
\hline No. solvent atoms & 89 \\
\hline Mean B-factor ( $\AA$ ) & 53.1 \\
\hline Rmsd bond lengths ( $\AA$ ) & 0.010 \\
\hline Rmsd bond angles ( $\left.{ }^{\circ}\right)$ & 1.04 \\
\hline Ramachandran statistics (\%) \\
\hline Favored & 98.3 \\
\hline Allowed & 1.7 \\
\hline Outlier & 0 \\
\hline PDB ID & $51 \mathrm{KW}$ \\
\hline Crystallization conditions & $10 \%(\mathrm{v} / \mathrm{v}) \mathrm{Broad} \mathrm{MW} \mathrm{PEG} \mathrm{smear,}^{3.2 \mathrm{M} \mathrm{MgCl}}, 100 \mathrm{mM} \mathrm{Hepes} \mathrm{pH}$ \\
& 7.0 \\
\hline
\end{tabular}

* Highest resolution shell shown in parentheses.

101 BIKE-1 crystals diffracted to $2.4 \AA$ and contained a single protein chain per asymmetric

102 unit (chain A). Sorrell and colleagues have recently reported the structural

103 characterization of all four NAK family member kinase domains, including BIKE and AAK1

104 [8]. As previously observed, our structure of BIKE displayed a catalytically-competent

105 conformation in which residues in the protein regulatory spine ("R spine"; residues M99,

106 Y111, H178 and F199) are aligned and side-chain atoms from conserved residues in $\beta 3$

107 and $\alpha-C$ (K79 and E95, respectively) form an ion pair $[14,15]$. Moreover, the NAK family-

108 exclusive helical domain (P209 to Y224) found C-terminal of the activation segment

109 (from ${ }^{198}$ DFG to APE ${ }^{233}$ domains), dubbed ASCH (activation segment C-terminal helix) [8,

110 16, 17], also displayed an identical conformation in the BIKE structure described here

\section{1 (Figure 1a).}


112 Figure 1. Crystallographic characterization of BIKE bound to 3-acylaminoindazole 1. (a)

113 BIKE-1 adopts an active conformation. Protein (cartoon) regions important for activity

114 and ligand interaction are indicated and highlighted in different colors. As for other

115 NAK-family members, the activation segment of BIKE (orange) contains a C-terminal

116 helical segment ( $\mathrm{ASCH}$; in red). Inhibitor 1 (carbon atoms in pink) and BIKE residues

117 (carbon atoms in yellow) taking part in the regulatory hydrophobic spine and the critical

118 ion pair leading to an active conformation are shown as sticks. (b) Details of the BIKE-1

119 interactions. Inhibitor $\mathbf{1}$ is anchored to the hinge (green ribbon) via hydrogen bonds

120 (dashed lines) and complements the hydrophobic cavity formed by aliphatic side chains

121 from $p$-loop (purple ribbon) and $\beta-6$ residues. Potential interactions between main chain

122 carbonyl and the cyclopropane group are indicated by dashed lines. Hydrogen bonds

123 between Asn185, Gln137 and the sulfonamide group are also shown (dashed lines). (c)

124 Superposition of NAK family proteins AAK1 (blue; PDB ID 4WSQ), BIKE (grey, PDB IDs

125 5IKW, this work), GAK (PDB ID 4Y8D) and MPSK1 (PDB ID 2BUJ) shows variation in the $p$ -

126 loop region despite good alignment in the hinge region. Compound anchoring hydrogen

127 bonds are shown for BIKE and 1 (sticks). (d) Electron density (2Fo-Fc) maps (grey mash;

$1281.0 \sigma$ contour) for 1 . (e) Primary sequence comparison of NAK family hinge and P-loop

129 regions.

130 The cocrystal structure of $\mathbf{1}$ and BIKE reveals that 3-acylaminoindazole $\mathbf{1}$ is anchored to

131 the protein hinge region (BIKE residues 131-137) via hydrogen bonds between the $\mathrm{N}$

132 atoms of the indazole group and main chain carbonyl and NH groups of residues G131

133 and C133, respectively. An additional hydrogen bond is made between the amino group 
134 of 1 and the carbonyl group of C133. At the other end of the inhibitor, the sulfonamide

135 group engages residues Q137 and N185 via hydrogen bonds. These interactions position

136 the pendant aliphatic group into a hydrophobic cavity within the P-loop formed by

137 residues $\mathrm{V} 65$ and $\mathrm{S} 65$. The indazole and the aniline rings are not coplanar to each other.

138 This flexibility provides excellent shape-complementarity between the inhibitor and the

139 ATP-binding pocket; especially with the aliphatic side chains from L187 and from p-loop

140 residues $L 57, A 58$ and $V 65$. There is also a sulfur- $\pi$ interaction between the aniline ring

141 and the sulfur atom of C197 (Figure 1b). Residue C197 is adjacent to the protein DFG

142 motif and has been proposed as a site of covalent modification by the inhibitor (5Z)-7-

143 oxozeaenol [8]. The cyclopropane group adjacent to the carboxyl group in $\mathbf{1}$ is

144 accommodated within a sharp turn of the peptide backbone involving residues

145 occupying positions $+3,+4$ and +5 from the so-called gatekeeper, or GK, residue (M130

146 in BIKE). This kink in the peptide backbone allows the carbonyl groups of residues GK+3

147 (C133) and GK+4 (R134) to point towards the cyclopropyl C-H group, approaching at a

148 distance of 3.6 and $3.3 \AA$, respectively (Figure 1b).

149 We compared our BIKE-1 structure with published structures of DDR1 (PDB: 5FDP),

150 BRAF (PDB: 4XV9), AAK1 (PDB: 4WSQ), and RIOK1 (PDB: 4OTP) to attempt to rationalize

151 the structural basis differential activity of $\mathbf{1}$ and $\mathbf{2}$ on some but not all kinases. Surface

152 electrostatic potential is quite different for kinases that show differential activity

153 compared with those that do not. AAK1, BIKE, and RIOK1 have bland surface charge

154 where the critical cyclopropyl moiety of $\mathbf{1}$ is accommodated within the binding pocket. 
155 In contrast, BRAF and DDR1 have highly charged surfaces around their

156 corresponding hinge binding regions.

157 Superimposing our BIKE-1 structure with the previously published structures from the

158 NAK family [8] by aligning the hinge residues ( $r$ sd $<0.3 \AA$ for 7 equivalent $\mathrm{C} \alpha$ ) revealed

159 that the P-loop of STK16 and GAK are substantially displaced compared to the position

160 occupied by this region in BIKE and AAK1 structures (Figure 1d). The extensive contacts

1611 makes with the P-loop may explain its selectivity for BIKE and AAK1 over the other two

162 members of the NAK family. Interestingly, the primary sequence alignment of NAK

163 family members reveals that sequence conservation is high for the P-loop region and

164 low for the hinge moiety (Figure 1e).

165 Computational studies. A range of computational methodologies was applied to shed

166 light on the differential affinities of 1 and 2. Energy minimization calculations in the

167 unbound state using the OPLS3 force field [18] and the GB/SA [19] solvation model

168 suggest that dehydration penalty upon binding is not a dominant factor, as similar

169 hydration values were obtained for $1(-29.8 \mathrm{kcal} / \mathrm{mol})$ versus $2(-29.2 \mathrm{kcal} / \mathrm{mol})$; the

170 cyclopropyl analog was actually slightly more penalized. To investigate the electronic

171 properties, electrostatic potential derived atomic charges using the B3LYP exchange-

172 correlation energy functional [20] and the $6-31 G^{* *}$ basis set [21] were obtained in the

173 gas phase. In this case, striking differences were observed for the $\mathrm{C}-\mathrm{H}$ bond adjacent to

174 the carbonyl group; it was much more polarized for cyclopropyl than isopropyl (Figure

175 2), with no major differences for the adjacent amide. 
176 Figure 2. Computationally generated electrostatic potential (ESP) derived atomic

177 charges for (A) 2 and (B) 1.

178 Beyond defining potential enthalpic differences between cyclopropyl and isopropyl

179 substituents, we also investigated possible entropic differences between $\mathbf{1}$ and $\mathbf{2}$ for

180 selected kinases. The Glide docking application was used to predict binding poses in

181 each investigated kinase, and the components of entropy change (translational,

182 rotational, and vibrational) upon binding of 1 and 2 to the kinases were calculated using

183 the RRHO approximation (Table 4). Translational and rotational entropic losses were

184 essentially equivalent for $\mathbf{1}$ and $\mathbf{2}$ at any given kinase. However, the vibrational entropic

185 gain was higher for 1 with all kinases except p38a. Interestingly, upon binding, the

186 largest overall changes in entropy, or $\Delta\left(\mathrm{T} \Delta \mathrm{S}_{\text {bind }}\right)$, between 1 and $\mathbf{2}$ were observed for

187 AAK1 and BIKE. These two kinases displayed the two largest $K_{d}$ ratios. $\Delta\left(T \Delta S_{\text {bind }}\right)$

188 between $\mathbf{1}$ and $\mathbf{2}$ was markedly lower for those kinases not displaying significant activity

189 cliffs. However, there are two exceptions to this observation, namely KIT and RIOK1. The

190 former displayed a significant $\Delta\left(T \Delta S_{b i n d}\right)$ between $\mathbf{1}$ and $\mathbf{2}$, although there is not a

191 significant difference in affinity. With RIOK1, the entropic difference between 1 and 2

192 was modest, despite a $K_{d}$ ratio 2:1 of 56 (Table 1).

193 Table 4. Thermodynamic parameters of binding 1 and 2 with kinases.

\begin{tabular}{|c|c|c|c|c|c|c|c|}
\hline \multirow{2}{*}{ Kinase } & \multirow{2}{*}{ PDB } & \multirow{2}{*}{ Cmpd } & \multicolumn{4}{|c|}{$\mathrm{T} \Delta S_{\text {bind }}$} & \multirow{2}{*}{$\Delta\left(\mathrm{T} \Delta \mathrm{S}_{\text {bind }}\right)$} \\
\hline & & & Trans & Rot & Vib & Total & \\
\hline \multirow{2}{*}{ AAK1 } & \multirow{2}{*}{$5 L 4 Q$} & 1 & -11.30 & -11.14 & 4.70 & -17.75 & \multirow{2}{*}{3.54} \\
\hline & & 2 & -11.31 & -11.07 & 1.09 & -21.29 & \\
\hline \multirow{2}{*}{ BIKE } & \multirow{2}{*}{$51 \mathrm{KW}$} & 1 & -11.30 & -11.07 & 4.64 & -17.73 & \multirow{2}{*}{2.51} \\
\hline & & 2 & -11.31 & -11.10 & 2.17 & -20.24 & \\
\hline
\end{tabular}




\begin{tabular}{|c|c|c|c|c|c|c|c|}
\hline \multirow{2}{*}{ p38a } & \multirow{2}{*}{ 5LAR } & 1 & -11.30 & -11.13 & 3.35 & -19.09 & \multirow{2}{*}{-0.10} \\
\hline & & 2 & -11.31 & -11.17 & 3.49 & -18.98 & \\
\hline \multirow{2}{*}{$A B L$} & \multirow{2}{*}{$4 Z O G$} & 1 & -11.30 & -11.05 & 3.15 & -19.20 & \multirow{2}{*}{1.64} \\
\hline & & 2 & -11.31 & -11.04 & 1.51 & -20.84 & \\
\hline \multirow{2}{*}{ KIT } & \multirow{2}{*}{$4 U 0 I$} & 1 & -11.30 & -11.13 & 3.09 & -19.34 & \multirow{2}{*}{3.23} \\
\hline & & 2 & -11.31 & -11.18 & -0.08 & -22.57 & \\
\hline \multirow{2}{*}{ DDR1 } & \multirow{2}{*}{ 5FDP } & 1 & -11.30 & -11.06 & 3.84 & -18.52 & \multirow{2}{*}{1.45} \\
\hline & & 2 & -11.31 & -11.08 & 2.41 & -19.97 & \\
\hline \multirow{2}{*}{ CSF1R } & \multirow{2}{*}{$4 \mathrm{R} 7 \mathrm{H}$} & 1 & -11.30 & -11.10 & 2.21 & -20.19 & \multirow{2}{*}{1.07} \\
\hline & & 2 & -11.31 & -11.10 & 1.14 & -21.27 & \\
\hline \multirow{2}{*}{ RIOK1 } & \multirow{2}{*}{ 40TP } & 1 & -11.30 & -11.03 & 6.66 & -15.68 & \multirow{2}{*}{0.64} \\
\hline & & 2 & -11.31 & -11.10 & 6.09 & -16.32 & \\
\hline \multirow{2}{*}{ RAF1 } & \multirow{2}{*}{$30 \mathrm{MV}$} & 1 & -11.30 & -11.06 & 2.82 & -19.54 & \multirow{2}{*}{1.52} \\
\hline & & 2 & -11.31 & -11.09 & 1.34 & -21.06 & \\
\hline
\end{tabular}

194

\section{DISCUSSION}

196 The term "activity cliff" has been proposed to describe pairs of structurally similar

197 compounds, like 1 and $\mathbf{2}$, with a large difference in potency relative to a common target

198 [22]. There have been other reports of kinase inhibitor activity cliffs involving

199 cyclopropyl and isopropyl carboxamides proximal to their hinge binding moiety (Table

200 5). The 2-cyclopropylcarboxamidopyridine 3a showed $>100 x$ higher affinity for TYK2

201 than the isopropyl analog $\mathbf{3 b}$ [23]. This effect is not limited to binding assays as

202 triazolopyrimidines 4 show $>100 x$ difference $I_{50}$ value in a JAK1 biochemical activity

203 assay [24]. Similarly, 5a displayed a much higher inhibition potency than $\mathbf{5 b}$ in an

204 enzymatic activity assay of mutant B-Raf ${ }^{\mathrm{V} 600 \mathrm{E}}$ activity [25]. These examples substantiate

205 our observations that subtle changes in structure can lead to selective inhibition of

206 specific protein kinases. To the best of our knowledge, 3-5 were not subjected to broad 
207 kinome activity characterization, and the breadth of the associated activity cliffs has not

208 been evaluated.

209 Table 5. Previously reported matched pairs of kinase inhibitors demonstrating an affinity

210 or activity cliff between cyclopropyl and isopropyl compounds.

211

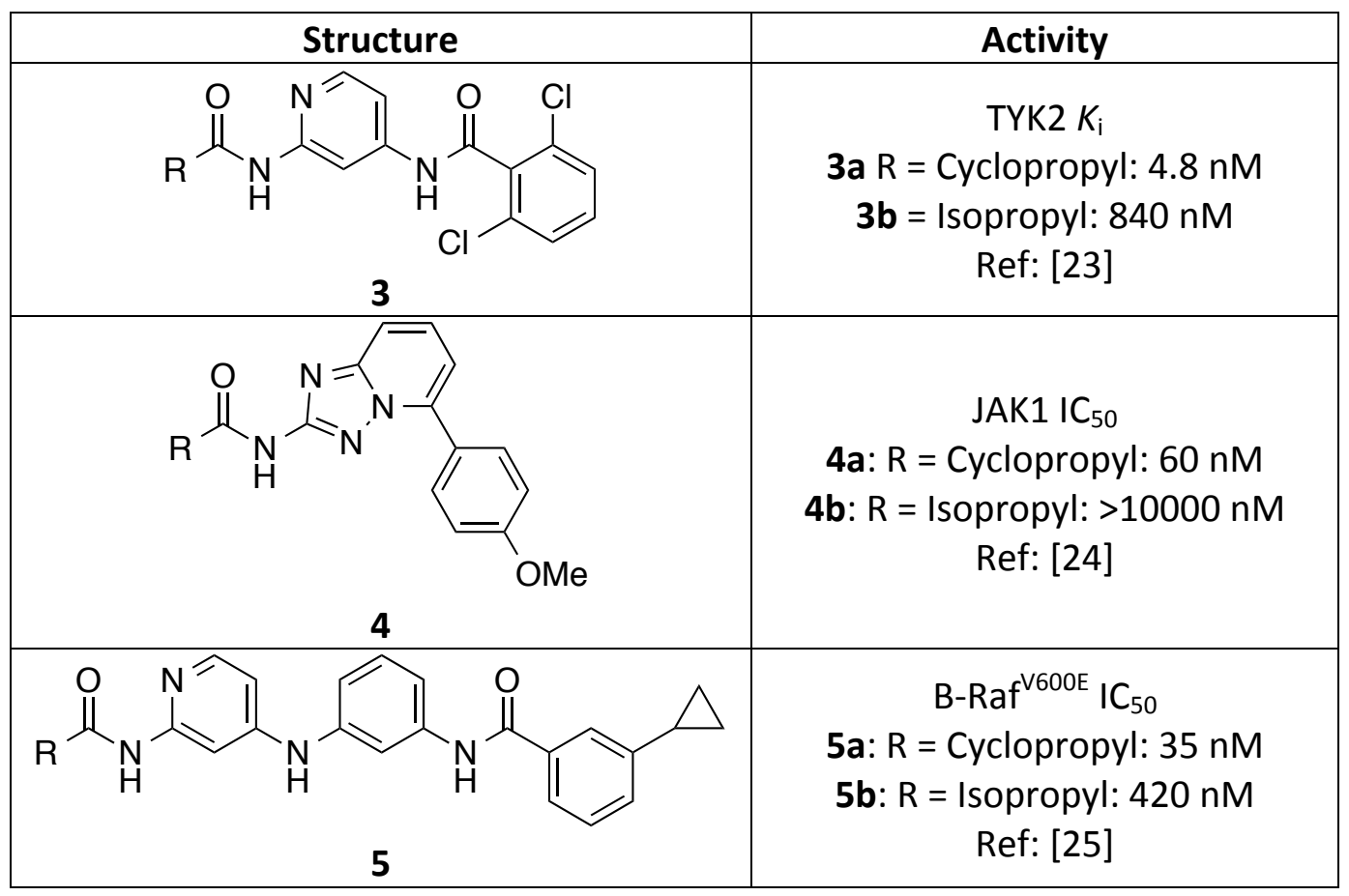

213 The cyclopropyl ring is a versatile lipophilic group that frequently appears in preclinical

214 and clinical candidate molecules. It has been described to have "magical" properties

215 that can enhance potency, reduce off-target effects, increase metabolic stability and

216 brain permeability, yet the precise molecular basis for these effects remains poorly

217 understood [26]. Theoretical studies on cyclopropane and its derivatives suggest that

218 atomic bonding results mainly from the overlap of three $s p^{2}$ hybrids (one on each

219 carbon) pointing towards the center of the ring. Thus, the electron distribution in the C- 
220 C internuclear region is not concentrated along the line between the nuclei, but rather

221 slightly outside this line. As a consequence, the C-C bonds have enhanced $\pi$ character,

222 and the $\mathrm{C}-\mathrm{H}$ bonds have more $\mathrm{s}$ character. In other words, the cyclopropyl ring behaves

223 much like an alkene, interacts with neighboring $\pi$-electron systems, and has $\mathrm{CH}$ bonds

224 with increased acidity relative to standard alkanes [27]. These effects are apparent

225 when the emerging negative charge on the $\mathrm{C}-\mathrm{H}$ carbon is stabilized by conjugation, such

226 as the one between the $\pi$-like cyclopropyl and an amide carbonyl group in 1. Calculated

227 electrostatic potential charges, as demonstrated above, may contribute to the activity

228 difference between 1 and $\mathbf{2}$.

229 3-Acylaminoindazoles are a known class of ATP-competitive protein kinase inhibitors

230 [28]. The heterocyclic core mimics the adenosine base of ATP and forms hydrogen

231 bonds with the hinge motif of the enzyme. 1 and 2, differing by only two hydrogen

232 atoms were screened across 400 human protein kinases to define both compounds as

233 narrow spectrum inhibitors, with high affinity binding to $<3 \%$ of the kinases surveyed.

234 Both 1 and 2 bound to seven kinases (ABL1, BRAF, DDR1, KIT, PDGFRA, PDGFRB, and

235 RAF1) with submicromolar affinity. Surprisingly, cyclopropyl amide $\mathbf{1}$ bound to an

236 additional four kinases (AAK1, BIKE, RIOK1, and RIOK3) where isopropyl amide 2 was not

237 effective. Thus, this pair of ATP-competitive kinase inhibitors have the potential to be

238 used to probe selectively the biology of just 4 protein kinases while controlling for over

239400 others in the human genome. 
240 BIKE and AAK1 are both members of the NAK (Numb-associated kinase) family of

241 protein kinases and share close sequence identity within their catalytic sites. Both

242 kinases are potently inhibited by the 3-acylaminoindazole $\mathbf{1}$ and are likely to interact

243 with the inhibitor through conserved interactions. The BIKE-1 cocrystal structure

244 provides a clear view into the molecular details of the enzyme-ligand interaction. The

245 critical cyclopropyl carboxamide sits in a pocket adjacent to the hinge-binding residues

246 and is orientated toward the protein surface. The amide $\mathrm{NH}$ of $\mathbf{1}$ and the cyclopropyl

247 ring $\mathrm{C}-\mathrm{H}$ sit in close proximity (2.70 and $3.29 \AA$, respectively) to the Cys133 carbonyl

248 oxygen (Figure 3). Our quantum mechanical calculations showed that the cyclopropyl

249 group has an increased polarization across the ring $\mathrm{C}-\mathrm{H}$ compared the corresponding

250 isopropyl analogue. The complex between the cyclopropyl derivative 1 and BIKE is

251 indicative of a $\mathrm{CH}-\mathrm{O}$ nonconventional hydrogen bond. Electrostatic interactions

252 dominate hydrogen bonds and the increased polarization of the cyclopropyl C-H bond,

253 as evidenced by the electrostatic potential charges, may explain why $\mathbf{1}$ shows

254 significantly higher affinity for AAK1 and BIKE than the isopropyl analog 2 but leaves

255 unanswered the question about why $\mathbf{1}$ and $\mathbf{2}$ are equipotent at other kinases.

256 Figure 3. (A) 1 bound in BIKE active site. (B) Two dimensional compound interaction

257 diagram of 1 and BIKE depicting adjacent residues and key interactions.

258 Modulating entropy contributions has previously been proposed as a means to increase

259 binding affinity between small molecules and proteins [29]. Upon binding, ligands lose

260 degrees of freedom with respect to translational, rotational, and vibrational motion. 
261 This loss of freedom constitutes a loss of entropy, which disfavors binding. Given its

262 triangular nature and smaller size, the cyclopropyl group may vibrate more freely within

263 the protein, which favor binding when compared to the isopropyl group. Importantly,

264 depending on the protein environment and architecture, this effect can be more

265 pronounced. In our cocrystal structure of BIKE and 1, the cyclopropyl group of interest is

266 more solvent exposed. As such, this group is not as restricted by the binding site and

267 does not experience a significant loss in vibrational entropy, which may be driving the

268 activity cliff observed between $\mathbf{1}$ and $\mathbf{2}$. Kinases for which an activity cliff between $\mathbf{1}$ and

2692 is not observed may have the cyclopropyl group less solvent exposed, leading to a

270 larger vibrational entropy loss.

271 We have suggested that differences in electrostatic potential or in vibrational entropy

272 changes may help to rationalize the activity cliffs between $\mathbf{1}$ and $\mathbf{2}$ in some kinases but

273 not in others. We recognize that there are myriad other subtle changes in the binding

274 pockets that must also be explored to enable prediction of which kinases can be

275 differentially inhibited by matched ligand pairs like $\mathbf{1}$ and $\mathbf{2}$. Our enhanced

276 understanding of the differential interactions of 1 and $\mathbf{2}$ within the binding pockets of

277 AAK1, BIKE, and other kinases will improve our ability to produce narrow spectrum

278 probes with optimal negative control analogs for use in biological studies.

\section{Supporting Information}

280 S1 Table. Results of KINOMEscan and select $K_{d}$ determinations for 1 and 2.

281 KINOMEscan assay panel and associated $K_{\mathrm{d}}$ determinations were conducted at 
282 DiscoverX Corporation. Compounds were evaluated as single measurements at $1 \mu \mathrm{M}$.

283 Displacement percentage ("Disp \%") reflects the extent to which the compound

284 displaced the kinase construct from an immobilized ligand. $K_{d}$ values were determined

285 for all kinases with $\geq 70 \%$ displacement with 1 in the KINOMEscan screen at $1 \mu \mathrm{M}$

286 compound and selected other kinases.

287 MATERIALS AND METHODS

$288 \mathrm{~N}$-(6-(3-(cyclopropanesulfonamido)phenyl)-1H-indazol-3-yl)cyclopropanecarboxamide

289 (1). ${ }^{1} \mathrm{H}$ NMR $\left(400 \mathrm{MHz}\right.$, Methanol- $\left.d_{4}\right) \delta 7.83-7.78(\mathrm{~d}, J=8.6 \mathrm{~Hz}, 1 \mathrm{H}), 7.61-7.54(\mathrm{~m}$, $2902 \mathrm{H}), 7.46-7.30(\mathrm{~m}, 3 \mathrm{H}), 7.29-7.23(\mathrm{ddd}, J=7.7,2.2,1.3 \mathrm{~Hz}, 1 \mathrm{H}), 2.62-2.49(\mathrm{tt}, J=8.0$,

$2914.8 \mathrm{~Hz}, 1 \mathrm{H}), 1.95-1.84(\mathrm{ddd}, J=12.5,7.9,4.2 \mathrm{~Hz}, 1 \mathrm{H}), 1.07-0.77(\mathrm{~m}, 8 \mathrm{H}) . \mathrm{MS}+(-\mathrm{ES}$

$292 \quad$ API) -397.1

$293 N$-(6-(3-(cyclopropanesulfonamido)phenyl)-1H-indazol-3-yl)isobutyramide (2). ${ }^{1} \mathrm{H}$ NMR

294 (400 MHz, Methanol- $\left.d_{4}\right) \delta 7.82-7.75(\mathrm{~d}, J=8.6 \mathrm{~Hz}, 1 \mathrm{H}), 7.62-7.54(\mathrm{dt}, J=10.9,1.5 \mathrm{~Hz}$, $2952 \mathrm{H}$ ), $7.47-7.31(\mathrm{~m}, 3 \mathrm{H}), 7.31-7.22(\mathrm{ddd}, J=7.8,2.2,1.3 \mathrm{~Hz}, 1 \mathrm{H}), 2.84-2.69$ (hept, $J=$ $2966.8 \mathrm{~Hz}, 1 \mathrm{H}), 2.61-2.50(\mathrm{tt}, J=8.0,4.8 \mathrm{~Hz}, 1 \mathrm{H}), 1.29-1.15(\mathrm{~d}, J=6.8 \mathrm{~Hz}, 6 \mathrm{H}), 1.14-0.82$ 297 (m, 4H). MS+ (- ES API) -399.1

298 DiscoverX kinase affinity measurements. KINOMEscan assay panel profiling and 299 associated $K_{\mathrm{d}}$ determinations were obtained at DiscoverX Corporation using their 300 previously described methodology [30, 31]. 
301 Binding displacement assays. Inhibitor binding was determined using a binding-

302 displacement assay, which tests the ability of the inhibitors to displace a fluorescent

303 tracer compound from the ATP binding site of the kinase domain. Inhibitors were

304 dissolved in DMSO and dispensed as 16-point, 2x serial dilutions in duplicate into black

305 multiwell plates (Greiner). Each well contained either $0.5 \mathrm{nM}$ or $1 \mathrm{nM}$ biotinylated

306 kinase domain protein ligated to streptavidin-Tb-cryptate (Cisbio), $12.5 \mathrm{nM}$ or $25 \mathrm{nM}$

307 Kinase Tracer 236 (ThermoFisher Scientific), 10 mM Hepes pH 7.5, 150 mM NaCl, 2 mM

$308 \mathrm{DTT}, 0.01 \% \mathrm{BSA}, 0.01 \%$ Tween-20. Final assay volume for each data point was $5 \mu \mathrm{L}$, and

309 final DMSO concentration was $1 \%$. The plate was incubated at room temperature for 1.5

310 hours and then read using a TR-FRET protocol on a PheraStarFS plate reader (BMG

311 Labtech). The data was normalized to $0 \%$ and $100 \%$ inhibition control values and fitted

312 to a four parameter dose-response binding curve in GraphPad Software. The

313 determined $I_{50}$ values were converted to $K_{\mathrm{i}}$ values using the Cheng-Prusoff equation

314 and the concentration and $K_{d}$ values for the tracer (previously determined).

315 Cloning, Expression, Purification and crystallization. BIKE $38-345$ (K320A, K321A) with a tobacco

316 etch virus (TEV) protease cleavable, $\mathrm{N}$-terminal 6xHis tag was expressed from vector

317 pNIC-ZB. To improve BIKE crystallization, a cluster of surface entropy reduction

318 mutations [32] was engineered into the expression construct, K320A/K321A [8]. For

319 protein production, the host $E$. coli strain, BL21(DE3)-R3 expressing lambda

320 phosphatase, was cultivated in TB medium $(+50 \mu \mathrm{g} / \mathrm{ml}$ kanamycin, $35 \mu \mathrm{g} / \mathrm{ml}$

321 chloramphenicol) at $37^{\circ} \mathrm{C}$ until $\mathrm{OD}_{600}$ reached $\sim 3$ and then cooled to $18^{\circ} \mathrm{C}$ for 1 hour.

322 Isopropyl 1-thio-D-galactopyranoside was added to $0.1 \mathrm{mM}$, and growth continued at 
$32318^{\circ} \mathrm{C}$ overnight. The cells were collected by centrifugation then resuspended in $2 x$ lysis

324 buffer (100 mM HEPES buffer, pH 7.5, $1.0 \mathrm{M} \mathrm{NaCl}, 20 \mathrm{mM}$ imidazole, $1.0 \mathrm{mM}$ tris(2-

325 carboxyethyl)phosphine, 2x Protease Inhibitors Cocktail Set VII (Calbiochem, 1/1000

326 dilution) and flash-frozen in liquid nitrogen. Cells were lysed by sonication on ice. The

327 resulting proteins were purified using Ni-Sepharose resin (GE Healthcare) and eluted

328 stepwise in binding buffer with $300 \mathrm{mM}$ imidazole. Removal of the hexahistidine tag was

329 performed at $4{ }^{\circ} \mathrm{C}$ overnight using recombinant TEV protease. Proteins were further

330 purified using reverse affinity chromatography on Ni-Sepharose followed by gel

331 filtration (Superdex 200 16/60, GE Healthcare). Protein in gel filtration buffer (25 mM

332 HEPES, $500 \mathrm{mM} \mathrm{NaCl}, 0.5 \mathrm{mM}$ TCEP, 5\% [v/v] glycerol) was concentrated to $12 \mathrm{mg} / \mathrm{ml}$

333 using $30 \mathrm{kDa}$ MWCO centrifugal concentrators (Millipore) at $4^{\circ} \mathrm{C}$. 3 -acylaminoindazole 1

334 in $100 \%$ DMSO was added to the protein in a 3 -fold molar excess and incubated on ice

335 for approximately 30 minutes. The mixture was centrifuged at 14,000 rpm for 10

336 minutes at $4^{\circ} \mathrm{C}$ prior to setting up $150 \mathrm{~nL}$ volume sitting drops at three ratios $(2: 1,1: 1$, or

337 1:2 protein-inhibitor complex to reservoir solution). Crystallization experiments were

338 performed at $20^{\circ} \mathrm{C}$. Crystals were cryoprotected in mother liquor supplemented with 20-

$33925 \%$ glycerol before flash-freezing in liquid nitrogen for data collection. Diffraction data

340 were collected at the Diamond Light Source. The best diffracting crystals grew under the

341 conditions described in Table 1 . When noted, crystal optimization used Newman's

342 buffer system [33] and defined PEG smears [34].

343 Structure Solution and Refinement. Diffraction data were integrated using XDS [35] and

344 scaled using AIMLESS from the CCP4 software suite [36]. Molecular replacement for was 
345 performed with Phaser [37] using BIKE/AZD7762 (PDB ID: 4W9W) [8] as a search model.

346 Automated model building was performed with Buccanner [38]. Automated refinement

347 was performed in PHENIX [39]. Coot [40] was used for manual model building and

348 refinement. Structure validation was performed using MolProbity [41]. Structure factors

349 and coordinates have been deposited in the PDB (see Table 1).

350 Computation. Solvation free energies and quantum mechanical calculations were run

351 using Macromodel ([42]) and Jaguar ([43]) from Schrodinger Inc. Further computational

352 studies were performed using Maestro 11 from Schrodinger (Schrödinger Release 2016-

353 4: Maestro, Schrödinger, LLC, New York, NY, 2016). The lowest energy configurations for

354 compounds 1 and $\mathbf{2}$ at $\mathrm{pH} 7$ were generated with the Ligand Preparation application

355 (Schrödinger Release 2016-4: LigPrep, Schrödinger, LLC, New York, NY, 2016). Next, PDB

356 crystal structures for AAK1, BIKE and the other kinases in Table 4 were downloaded and

357 prepared for docking. Briefly, bond orders were assigned, hydrogen atoms were added,

358 selenomethionine residues were converted to methionines, and missing side chains

359 were filled with the Prime application. The protein grid for docking was set by selecting

360 the center of the cocrystal ligand in the PDB structure. The Glide application was used

361 for docking of 1 and 2 to all kinases (Schrödinger Release 2016-4: Glide, Schrödinger,

362 LLC, New York, NY, 2016.). Flexible ligand sampling was allowed with the XP (extra

363 precision) scoring function (http://pubs.acs.org/doi/abs/10.1021/jm0512560). Once the

364 ligands were docked and the lowest energy poses were retrieved, a Rigid Rotor

365 Harmonic Oscillation (RRHO) approximation was performed on the theoretical protein-

366 ligand complex (https://www.ncbi.nlm.nih.gov/pmc/articles/PMC3329805/). For the 
367 RRHO approximation, an OPLS_2005 force field was applied

368 (http://pubs.acs.org/doi/abs/10.1021/acs.jctc.5b00864).

\section{ACKNOWLEDGMENT}

370 Aled Edwards is acknowledged for illuminating discussions and encouragement in the

371 preparation of this manuscript. We thank Diamond Light Source for access to beamline

372103 (proposal number MX14664) that contributed to the results presented here. The

373 SGC is a registered charity (number 1097737) that receives funds from AbbVie, Bayer

374 Pharma AG, Boehringer Ingelheim, Canada Foundation for Innovation, Eshelman

375 Institute for Innovation, Genome Canada, Innovative Medicines Initiative (EU/EFPIA),

376 Janssen, Merck \& Co., Novartis Pharma AG, Ontario Ministry of Economic Development

377 and Innovation, Pfizer, São Paulo Research Foundation-FAPESP, Takeda, and Wellcome

378 Trust. 
bioRxiv preprint doi: https://doi org/10.1101/094631; this version posted March 2 2017. The copyright holder for this preprint (which was not certified by peer review) is the author/funder, who has granted bioRxiv a license to display the preprint in perpetuity. It is made available under aCC-BY-NC-ND 4.0 International license.

\section{$380 \quad$ Figure 1.}

a

C

381

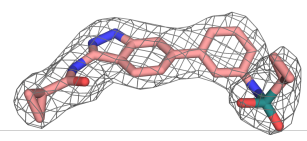

b

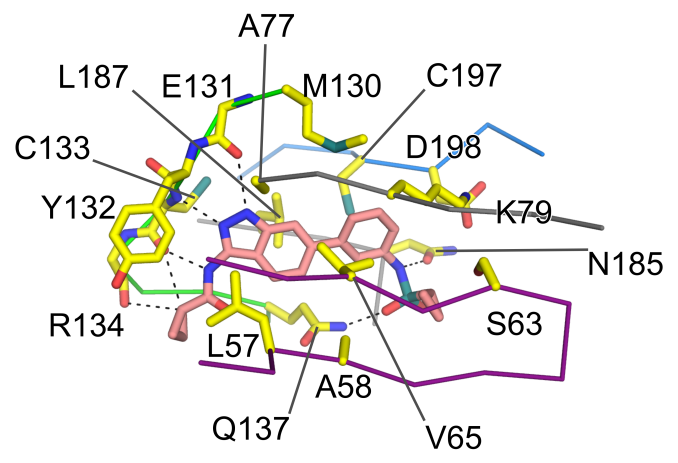

hinge

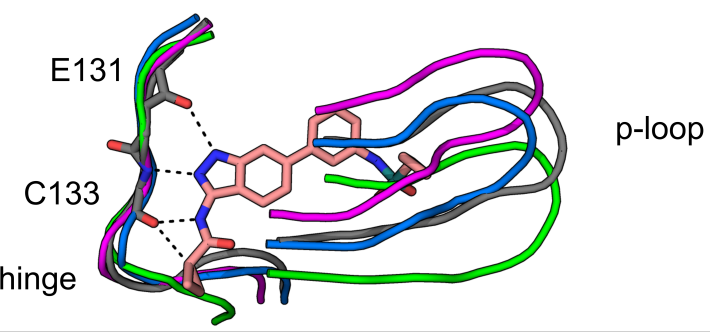


bioRxiv preprint doi: https://doi.org/10.1101/094631; this version posted March 2,2017 . The copyright holder for this preprint (which was not certified by peer review) is the author/funder, who has granted bioRxiv a license to display the preprint in perpetuity. It is made available under aCC-BY-NC-ND 4.0 International license.

\section{$384 \quad$ Figure 2.}

A

385<smiles>CN(C(=O)[C@H](O)C(C)(C)C)c1n[nH]c2cc(-c3cccc(NS(=O)(=O)C4CC4)c3)ccc12</smiles>

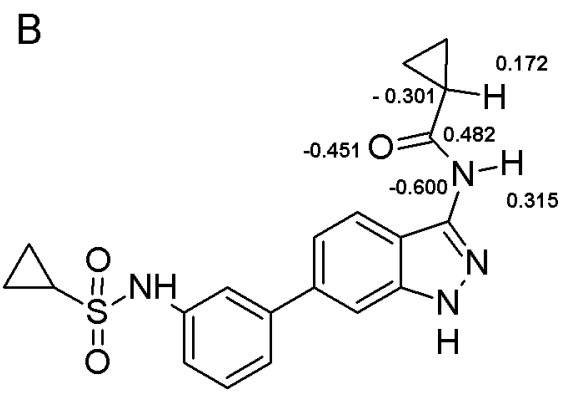

\section{6}


bioRxiv preprint doi: https://doi.org/10.1101/094631; this version posted March 2, 2017. The copyright holder for this preprint (which was not certified by peer review) is the author/funder, who has granted bioRxiv a license to display the preprint in perpetuity. It is made available under aCC-BY-NC-ND 4.0 International license.

\section{$387 \quad$ Figure 3.}

A

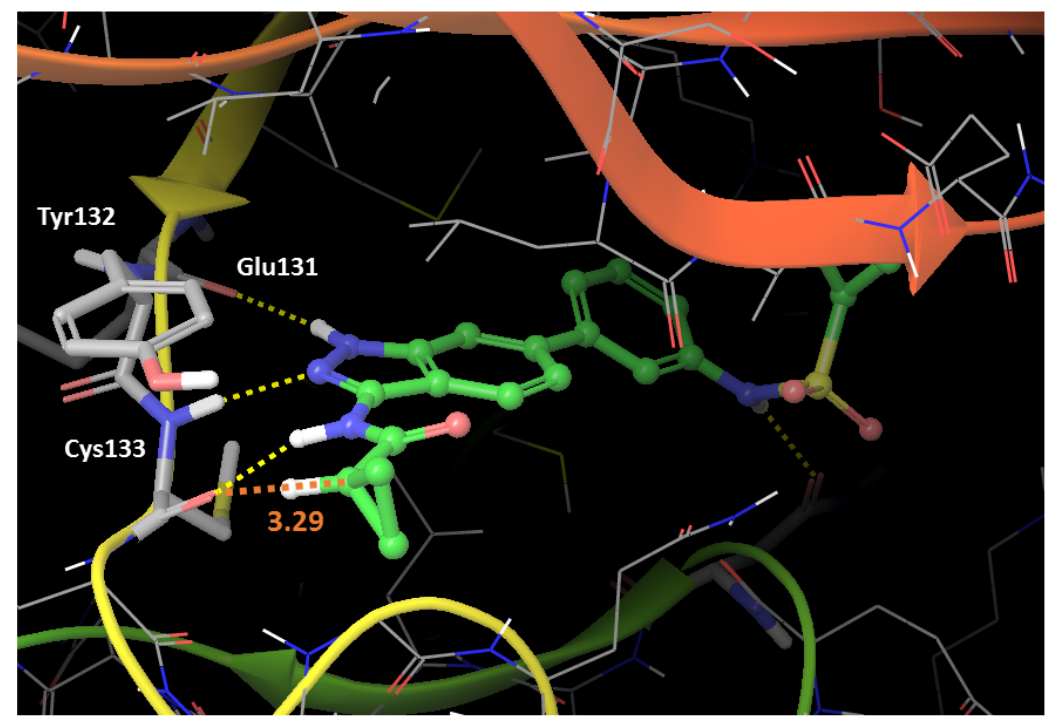

B

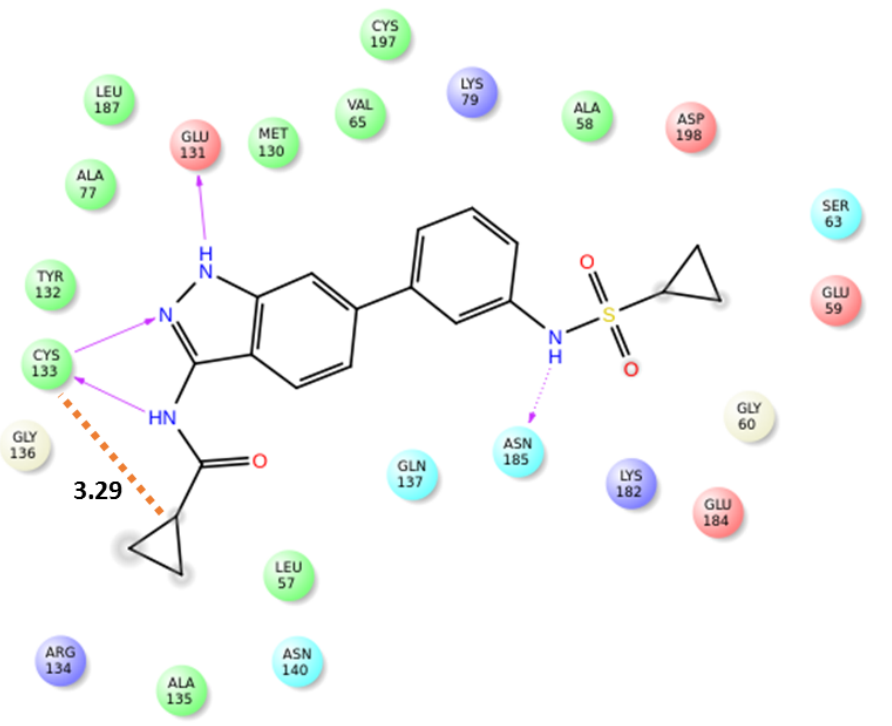




\section{REFERENCES}

$390 \quad 1 . \quad$ Cohen P, Alessi DR. Kinase drug discovery--what's next in the field? ACS Chem

391 Biol. 2013;8(1):96-104. doi: 10.1021/cb300610s. PubMed PMID: 23276252; PubMed 392 Central PMCID: PMCPMC4208300.

3932 2. Fedorov O, Muller S, Knapp S. The (un)targeted cancer kinome. Nat Chem Biol. 394 2010;6(3):166-9. doi: 10.1038/nchembio.297. PubMed PMID: 20154661.

395 3. Uitdehaag JC, Verkaar F, Alwan H, de Man J, Buijsman RC, Zaman GJ. A guide to 396 picking the most selective kinase inhibitor tool compounds for pharmacological 397 validation of drug targets. Br J Pharmacol. 2012;166(3):858-76. doi: 10.1111/j.1476398 5381.2012.01859.x. PubMed PMID: 22250956; PubMed Central PMCID: PMCPMC3417414.

400 4. Ruegg UT, Burgess GM. Staurosporine, K-252 and UCN-01: potent but nonspecific inhibitors of protein kinases. Trends Pharmacol Sci. 1989;10(6):218-20. PubMed PMID: 2672462.

403 5. Knapp S, Arruda P, Blagg J, Burley S, Drewry DH, Edwards A, et al. A public404 private partnership to unlock the untargeted kinome. Nat Chem Biol. 2013;9(1):3-6. doi: 405 10.1038/nchembio.1113. PubMed PMID: 23238671.

6. Arrowsmith $\mathrm{CH}$, Audia JE, Austin C, Baell J, Bennett J, Blagg J, et al. The promise and peril of chemical probes. Nat Chem Biol. 2015;11(8):536-41. doi: 10.1038/nchembio.1867. PubMed PMID: 26196764.

409 7. Xing L, Klug-Mcleod J, Rai B, Lunney EA. Kinase hinge binding scaffolds and their 410 hydrogen bond patterns. Bioorg Med Chem. 2015;23(19):6520-7. doi: 10.1016/j.bmc.2015.08.006. PubMed PMID: 26358279.

412 8. Sorrell FJ, Szklarz M, Abdul Azeez KR, Elkins JM, Knapp S. Family-wide Structural 413 Analysis of Human Numb-Associated Protein Kinases. Structure. 2016;24(3):401-11. doi: 10.1016/j.str.2015.12.015. PubMed PMID: 26853940; PubMed Central PMCID: PMCPMC4780864. BMP2K contributes to high myopia. J Clin Lab Anal. 2009;23(6):362-7. doi: 10.1002/jcla.20344. PubMed PMID: 19927351. associated protein kinase 1 and cyclin G-associated kinase regulate hepatitis $C$ virus entry and are potential drug targets. J Virol. 2015;89(8):4387-404. doi: 10.1128/JVI.02705-14. PubMed PMID: 25653444; PubMed Central PMCID: PMC4442395.

424 11. Sakurai MA, Ozaki Y, Okuzaki D, Naito Y, Sasakura T, Okamoto A, et al. Gefitinib and luteolin cause growth arrest of human prostate cancer PC-3 cells via inhibition of cyclin G-associated kinase and induction of miR-630. PLoS One. 2014;9(6):e100124. doi: 10.1371/journal.pone.0100124. PubMed PMID: 24971999; PubMed Central PMCID: PMCPMC4074034.

429 12. Shi B, Conner SD, Liu J. Dysfunction of endocytic kinase AAK1 in ALS. Int J Mol Sci. 430 2014;15(12):22918-32. doi: 10.3390/ijms151222918. PubMed PMID: 25514244; 431 PubMed Central PMCID: PMCPMC4284746. 
432 13. Lebakken CS, Riddle SM, Singh U, Frazee WJ, Eliason HC, Gao Y, et al. 433 Development and applications of a broad-coverage, TR-FRET-based kinase binding assay 434 platform. J Biomol Screen. 2009;14(8):924-35. doi: 10.1177/1087057109339207. 435 PubMed PMID: 19564447.

436 14. Huse M, Kuriyan J. The conformational plasticity of protein kinases. Cell. 2002;109(3):275-82. PubMed PMID: 12015977.

438 15. Kornev AP, Taylor SS. Defining the conserved internal architecture of a protein kinase. Biochim Biophys Acta. 2010;1804(3):440-4. doi: 10.1016/j.bbapap.2009.10.017. PubMed PMID: 19879387; PubMed Central PMCID: PMCPMC3435107. nanobodies. Biochem J. 2014;459(1):59-69. doi: 10.1042/BJ20131399. PubMed PMID: 24438162 ; PubMed Central PMCID: PMCPMC3957475.

17. Eswaran J, Bernad A, Ligos JM, Guinea B, Debreczeni JE, Sobott F, et al. Structure of the human protein kinase MPSK1 reveals an atypical activation loop architecture. Structure. 2008;16(1):115-24. doi: 10.1016/j.str.2007.10.026. PubMed PMID: 18184589; PubMed Central PMCID: PMCPMC2194165. Field Providing Broad Coverage of Drug-like Small Molecules and Proteins. J Chem Theory Comput. 2016;12(1):281-96. doi: 10.1021/acs.jctc.5b00864. PubMed PMID: 26584231.

453 19. Still WC, Tempczyk A, Hawley RC, Hendrickson T. Semianalytical Treatment of Solvation for Molecular Mechanics and Dynamics. J Am Chem Soc. 1990;112:6127-9.

22. Stumpfe D, Bajorath J. Exploring activity cliffs in medicinal chemistry. J Med Chem. 2012;55(7):2932-42. doi: 10.1021/jm201706b. PubMed PMID: 22236250. identification of novel and selective TYK2 inhibitors. Eur J Med Chem. 2013;67:175-87. doi: 10.1016/j.ejmech.2013.03.070. PubMed PMID: 23867602.

24. Menet CJ, Fletcher SR, Van Lommen G, Geney R, Blanc J, Smits K, et al. Triazolopyridines as selective JAK1 inhibitors: from hit identification to GLPG0634. J Med Chem. 2014;57(22):9323-42. doi: 10.1021/jm501262q. PubMed PMID: 25369270. aminopyridin-2-yl)amides as B-Raf(V600E) inhibitors. Bioorg Med Chem Lett. 2016;26(12):2760-3. doi: 10.1016/j.bmcl.2016.04.076. PubMed PMID: 27155899. Appears in Preclinical/Clinical Drug Molecules. J Med Chem. 2016;59(19):8712-56. doi: 10.1021/acs.jmedchem.6b00472. PubMed PMID: 27299736.

27. Galano A, Alvarez-Idaboy J, Vivier-Bunge A. Non-alkane behavior of cyclopropane and its derivatives: characterization of unconventional hydrogen bond interactions. 473 Theor Chem Account. 2007;118:597-606.

474 28. Witherington J, Bordas V, Gaiba A, Naylor A, Rawlings AD, Slingsby BP, et al. 6475 heteroaryl-pyrazolo[3,4-b]pyridines: potent and selective inhibitors of glycogen 
476 synthase kinase-3 (GSK-3). Bioorg Med Chem Lett. 2003;13(18):3059-62. PubMed PMID:

47712941333.

478 29. Chang CE, Chen W, Gilson MK. Ligand configurational entropy and protein

479 binding. Proc Natl Acad Sci U S A. 2007;104(5):1534-9. doi: 10.1073/pnas.0610494104.

480 PubMed PMID: 17242351; PubMed Central PMCID: PMCPMC1780070.

481 30. Davis MI, Hunt JP, Herrgard S, Ciceri P, Wodicka LM, Pallares G, et al. 482 Comprehensive analysis of kinase inhibitor selectivity. Nat Biotechnol. 483 2011;29(11):1046-51. doi: 10.1038/nbt.1990. PubMed PMID: 22037378.

484 31. Fabian MA, Biggs WH, 3rd, Treiber DK, Atteridge CE, Azimioara MD, Benedetti $485 \mathrm{MG}$, et al. A small molecule-kinase interaction map for clinical kinase inhibitors. Nat Biotechnol. 2005;23(3):329-36. doi: 10.1038/nbt1068. PubMed PMID: 15711537.

32. Derewenda ZS. Rational protein crystallization by mutational surface engineering. Structure. 2004;12(4):529-35. doi: 10.1016/j.str.2004.03.008. PubMed PMID: 15062076.

33. Newman J. Novel buffer systems for macromolecular crystallization. Acta crystallographica Section D, Biological crystallography. 2004;60(Pt 3):610-2. doi: 10.1107/S0907444903029640. PubMed PMID: 14993709.

34. Chaikuad A, Knapp S, von Delft F. Defined PEG smears as an alternative approach to enhance the search for crystallization conditions and crystal-quality improvement in reduced screens. Acta crystallographica Section D, Biological crystallography. 2015;71(Pt 8):1627-39. doi: 10.1107/S1399004715007968. PubMed PMID: 26249344; PubMed Central PMCID: PMC4528798.

35. Kabsch W. Xds. Acta crystallographica Section D, Biological crystallography. 2010;66(Pt 2):125-32. doi: 10.1107/S0907444909047337. PubMed PMID: 20124692; PubMed Central PMCID: PMC2815665.

501 36. Winn MD, Ballard CC, Cowtan KD, Dodson EJ, Emsley P, Evans PR, et al. Overview of the CCP4 suite and current developments. Acta crystallographica Section D, Biological crystallography. 2011;67(Pt 4):235-42. doi: 10.1107/S0907444910045749. PubMed PMID: 21460441; PubMed Central PMCID: PMC3069738.

505 37. McCoy AJ, Grosse-Kunstleve RW, Adams PD, Winn MD, Storoni LC, Read RJ. Phaser crystallographic software. Journal of applied crystallography. 2007;40(Pt 4):65874. doi: 10.1107/S0021889807021206. PubMed PMID: 19461840; PubMed Central PMCID: PMC2483472.

509

38. Cowtan K. The Buccaneer software for automated model building. 1. Tracing 9):1002-11. doi: 10.1107/S0907444906022116. PubMed PMID: 16929101.

39. Zhang KY, Cowtan K, Main P. Combining constraints for electron-density modification. Methods in enzymology. 1997;277:53-64. PubMed PMID: 18488305. PMC2852313. 
bioRxiv preprint doi: https://doi org/10.1101/094631; this version posted March 2 2017. The copyright holder for this preprint (which was not certified by peer review) is the author/funder, who has granted bioRxiv a license to display the preprint in perpetuity. It is made available under aCC-BY-NC-ND 4.0 International license.

520 crystallographica Section D, Biological crystallography. 2010;66(Pt 1):12-21. doi: 521 10.1107/S0907444909042073. PubMed PMID: 20057044; PubMed Central PMCID: 522 PMC2803126.

523 42. Schrödinger Release 2016-3: MacroModel, Schrödinger, LLC New York, NY, 2016.

524 43. Schrödinger Release 2016-4: Jaguar, Schrödinger, LLC, New York, NY, 2016.

525

526 